\title{
mission control
}

\section{ALMA's long look}

\section{ALMA's Band 1 receivers will open up the $7 \mathrm{~mm}$ window to the 66 antennas on Chajnantor Plateau. Oscar Morata and Ted Huang relate the expected delivery schedule and science goals for these instruments.}

$\mathrm{T}$ he Atacama Large Millimeter/ submillimeter Array (ALMA) is an interferometer composed of fifty $12-\mathrm{m}$ antennas in its main array, as well as four 12-m and twelve 7-m antennas in its compact array (the Atacama Compact Array, ACA). These dishes are all located on the Chajnantor Plateau in northern Chile at a very high altitude of $5,000 \mathrm{~m}$. ALMA is the pre-eminent astronomical imaging and spectroscopic instrument at $\mathrm{mm} / \mathrm{sub}-\mathrm{mm}$ wavelengths for the foreseeable future. The original ALMA design defined ten frequency bands that would cover the range from 30 to $1,000 \mathrm{GHz}$ and currently the instrument offers eight frequency bands (Bands 3-10) for general observations. Band 5 is the latest addition ${ }^{1}$ and Band 2 development is currently in an early phase.

The ALMA Band 1 receiver will fulfil the lower frequency range requirements of ALMA with the goal of giving access to frequencies of $35-50 \mathrm{GHz}(\sim 7 \mathrm{~mm}$ wavelengths) with the superior angular resolution and sensitivity that ALMA offers. An extension of up to $52 \mathrm{GHz}$ is available on a best-effort basis. The Band 1 bandwidth to central frequency ratio is the largest for any ALMA receiver band $(\sim 36 \%)$, and the range will include several astrophysically important spectral lines, such as the CS $(J=1-0)$ line at $49.0 \mathrm{GHz}$, the $\mathrm{SiO}(J=1-0)$ line at $43.4 \mathrm{GHz}$ and several $\mathrm{CH}_{3} \mathrm{OH}$ lines.

As a general-purpose instrument, the ALMA Band 1 receiver has a large number of varied science goals ${ }^{2}$. The two main science goals are: (1) the detection of molecular line emission from intermediate- $(z \sim 1.5)$ to highredshift $(6<z<10)$ galaxies using $\mathrm{CO}$ lines, allowing the detection of molecular gas from the peak of star formation up to the era of reionization; and (2) the study of the evolution of dust-grain growth from $\mathrm{mm}$ - to $\mathrm{cm}$-sized pebbles in protoplanetary disks, which will help in the localization and characterization of the

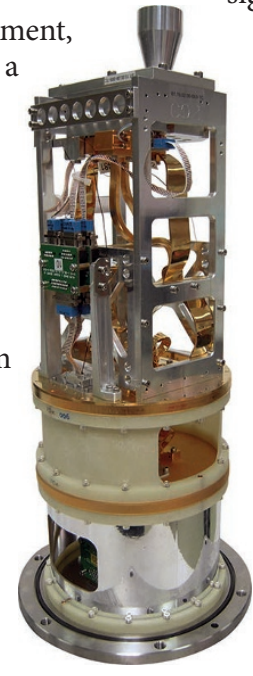

coagulation processes of dust grains that eventually lead to the formation of planets.

The wider gamut of scientific cases for Band 1 includes solar studies; the chemical differentiation of gas in starless cores in molecular clouds; the detection of complex molecules and the formation pathways of biomolecules such as amino acids and sugars; the study of the magnetic fields in star-forming regions through the detection of the Zeeman effect; the detection of the very curious emission from very small grains; the detection of $\mathrm{CH}_{3} \mathrm{OH}$ and $\mathrm{SiO}$ maser emission in young stellar objects and evolved stars; the study of the structure of clusters of galaxies using the Sunyaev-Zel'dovich effect; and so on.

The Band 1 receiver is a dual-polarization, single side-band heterodyne receiver with an intermediate frequency band of $4-12 \mathrm{GHz}$ (Fig. 1) $)^{3}$. The receiver consists of three subsystems: optics, cold (15 K) cartridge and warm $(300 \mathrm{~K})$ cartridge. The incoming beam from the ALMA antenna is refocused by a warm lens and collected by a corrugated aluminium feed horn at the $15 \mathrm{~K}$ stage. The two orthogonal polarizations of light are then split using an orthomode transducer and the signals are subsequently amplified with heterojunction field-effect-transistor cryogenic low-noise amplifiers fixed at the $15 \mathrm{~K}$ stage. The signal is then further amplified in the second stage by commercially available Q-band amplifiers at $300 \mathrm{~K}$. The upper side-band configuration is realized by using a high-pass filter after the Q-band amplifier. The sensitivity of the receiver is mainly determined by the noise and gain performance of the cryogenic low-noise amplifiers. The technical requirements for the Band 1 receivers are far more stringent than for any existing system at this frequency band. The specifications call for a receiver noise temperature of less than $25 \mathrm{~K}$ for $80 \%$ of the band, and less than $32 \mathrm{~K}$ for the entire band.

The plan is to assemble and deliver 73 receiver units to be installed in all 66 ALMA antennas, including spares. Currently, the ALMA Band 1 Receiver
Development Project is entering the production phase. Three receiver units are already assembled, and ten are expected by the end of 2017. After that, production will ramp up to about two receiver units per month. The plans are to install all of the receiver units at ALMA by early 2020 with the goal of offering the instrument to the community for ALMA observing Cycle 8 in April 2020. Band 1 Science Verification observations are expected to be performed during 2018/2019 and delivered to the community by late 2019 .

The Band 1 receiver is expected to reach a 1-hour point source observation r.m.s. sensitivity of 5.4-6.2 $\mu \mathrm{Jy}$ per beam in continuum and $\sim 0.4-0.8$ mJy per beam in spectral line observations. ALMA with Band 1 will be as sensitive or better, in this frequency range, as the Karl G. Jansky Very Large Array for point source detection, but will have a much higher mapping speed. The superior atmospheric transmission of the Chajnantor Plateau in this frequency range and its very low dependence on the precipitable water-vapour content of the atmosphere will allow the use of the Band 1 receiver in almost all weather conditions, year-round.

The ALMA Band 1 receivers are the ALMA East Asia contribution to ALMA development. The Development Project is a consortium led by the Academia Sinica Institute of Astronomy and Astrophysics (ASIAA) in Taiwan, in collaboration with the National Astronomical Observatory of Japan (NAOJ), the Universidad de Chile, the National Research Council of Canada Herzberg Institute and the National Radio Astronomy Observatory (NRAO).

\section{OSCAR MORATA and TED HUANG} are at the Academia Sinica Institute of Astronomy and Astrophysics, PO Box 23-141, Taipei 10617, Taiwan. e-mail: omorata@asiaa.sinica.edu.tw

\section{References}

1. Humphreys, L. et al. The Messenger 167, 7-10 (2017).

2. Di Francesco, J. et al. Preprint at https://arxiv.org/abs/1310.1604 (2013).

3. Huang, Y. D. et al. Proc. SPIE 9911, 99111 V (2016).

\section{Additional information}

Publisher's note: Springer Nature remains neutral with regard to jurisdictional claims in published maps and institutional affiliations. 